\title{
COMPARISON OF AVAILABILITY OF GALILEO, GPS AND A COMBINED GALILEO/GPS NAVIGATION SYSTEMS
}

\author{
Jiexian Wang \\ Department of Surveying Engineering, Tongji University, P.R. of China \\ Wolfgang Keller and Mohammad A. Sharifi \\ Department of Geodesy and GeoInformatics, University of Stuttgart \\ Geschwister-Scholl-Str. 24/D, D-70174 Stuttgart, Germany \\ e-mail: Sharifi@gis.uni-stuttgart.de
}

\begin{abstract}
Galileo satellites have been planned to be launched in 2006 by the European Union. Launching these satellites will double the number of positioning satellites currently accessible to users. As a consequence, accuracy and reliability will be improved dramatically. Besides accuracy and reliability, availability is also used for quantification of a Global Navigation Satellite System performance. This paper briefly describes the constellation of space segment of the proposed Galileo navigation system in comparison with the well-known Global Positioning System (GPS). Availability of GPS, Galileo and Galileo/GPS satellites are then formulated in terms of Satellite Appearance Distribution Function (SADF). Achievable accuracy in horizonal and vertical positions and timing are expressed using the respective Dilution of Precisions based on the derived mathematical formulation.
\end{abstract}

\section{INTRODUCTION}

Satellite navigation is going to play a key role in everyday life in the near future. The existing Global Navigation Satellite Systems (GNSS), GPS as the US type and GLONASS as the Russian one, have already demonstrated GNSS efficiency to land, sea, and air users. They were originally designed for military use and are still under national control. Although the number of civil users is higher than 100 millions, some severe shortcomings such as the limited achievable accuracy, missing guarantee for service, lack of signal integrity are evident for the civil users (Wolfrum and Trautenberg, 2000). This situation has been an advantageous motivation for establishing a civil GNSS with a global coverage.

As the pioneer, the European Union together with the European Space Agency are developing a new generation of (GNSS) called Galileo. Its definition phase has started in 1999 and ended in 2001 with defining the initial requirements and the system architecture. The design and validation phase, from 2002 to 2005, is comprised of consolidation of the requirements, the satellites and ground base components development and in-orbit validation (Seeber, 2003). Up to four satellites will be launched in the initial deployment 
(2004-2006). Finally, launching the remaining satellites by 2008 will make the system fully operational.

Galileo consists of $30(27+3$ active spares) satellites equally spaced in three circular Medium Earth Orbits (MEO). Being a global system from the beginning is the rationale for using the satellites of the MEO type. The orbital altitude is $23616 \mathrm{~km}$ and the inclination angle is $56^{\circ}$. The satellites move around the earth every 14 hours in the orbital planes and the ground tracks repeat every 10 days.

On the one hand, Galileo will be complementary to GPS and on the other hand, interoperable with it. Therefore, the overall GNSS will remedy the existing shortcoming and make it more robust and reliable. Indeed, performing control of the system by civil authorities will remove the limits in the achievable accuracy.

For technical comparison of Galileo and GPS performance, we should compare their performance measures. In the context of GNSS, availability, accuracy, continuity and integrity are mostly used as the quantification parameters of a GNSS performance.

In this paper, we will compare availability of two systems. To avoid confusion due to existence of several different definitions, let us define the measure first. According to US Federal Radionavigation Plan (FRP), the availability of a navigation system is the percentage of time that the services of the system are within the required performance limits (FRP, 2001). Alternatively, availability refers to the number of satellites available to the user. This is the definition used by many authors (see, e.g., O'Keefe et al., 2002; Wolfrum and Trautenberg, 2000; Wang et al., 2002). Herein, we also consider the later definition of the measure.

As already mentioned, instead of $24 \mathrm{MEO}$ satellites in GPS system Galileo is composed of 30 satellites. Consequently, the global availability is increased and as a by-product, the geometric situation is also improved. Eventually, from availability point of view, deployment of the Galileo satellites will introduce a high performance satellite-based navigation.

Herein, we introduce an innovative approach for the MEO satellites availability computation. Besides developing the mathematical formulation of the method, different achievable accuracy using GPS, Galileo and a combined Galileo/GPS are compared.

As discussed in Santerre R. (1991), since the satellites' inclinations are different from $90^{\circ}$ the satellites sky distribution is a function of latitude $\phi$. To clarify the dependency, the aforementioned accuracy indices are visualized as functions of site latitude.

\section{SATELLITE APPEARANCE DISTRIBUTION FUNCTION (SADF)}

For a satellite moving along azimuth $\alpha$ and geocentric latitude $\phi$ in a celestial frame, trajectory of the satellite can be parameterized using the geodesic equation on the mean sphere of the orbit, $C(0, R)$, as follows (e.g., Krakiwsky and Thomson, 1974)

$$
R \cos \phi \sin \alpha=c,
$$

where $c$ is a constant. This constant value can be expressed as a function of $R$ and $\alpha$ at ascending node, $\phi=0$

$$
R \sin \alpha_{0}=c,
$$

where $\alpha_{0}$ is the satellite's azimuth at ascending node (complement of the orbit inclination $i$ ). For instance, it is equal to $35^{\circ}$ and $34^{\circ}$ for GPS and Galileo satellites respectively. Substituting eq. (2) into eq. (1) is recast into

$$
\cos \phi \sin \alpha=\cos i \text {. }
$$


Decomposition of the satellite angular velocity $\omega$, to the meridian component $\omega_{\phi}$ and the parallel one $\omega_{\lambda}$, as the respective N-S and E-W direction velocities yields

$$
\left\{\begin{array}{l}
\omega_{\phi}=\frac{2 \pi R}{T} \frac{\cos \alpha}{R}=\frac{2 \pi}{T} \cos \alpha=\frac{2 \pi}{T} \sqrt{1-\frac{\cos ^{2} i}{\cos ^{2} \phi}} \\
\omega_{\lambda}=\frac{2 \pi R}{T} \frac{\sin \alpha}{R \cos \phi}=\frac{2 \pi}{T} \frac{\sin \alpha}{\cos \phi}=\frac{2 \pi}{T} \frac{\cos i}{\cos ^{2} \phi}
\end{array}\right.
$$

$T$ is the orbital period of the satellites which is equal to $11^{h} 56^{m}$ and $14^{h} 22^{m}$ for GPS and Galileo satellites respectively. Obviously, ground stations experience the satellite angular velocity along the $\lambda$ direction less than the real value due to the earth rotation and retrograde motion (in the same direction as the earth rotation, $0^{\circ} \leq i \leq 90^{\circ}$ ) of the satellites. So, the relative angular velocity components of the satellites $\left(\omega_{\phi}^{r}, \omega_{\lambda}^{r}\right)$, to an observer on the Earth are

$$
\left\{\begin{array}{l}
\omega_{\phi}^{r}=\frac{2 \pi}{T} \sqrt{1-\frac{\cos ^{2} i}{\cos ^{2} \phi}} \\
\omega_{\lambda}^{r}=\frac{2 \pi}{T} \frac{\cos i}{\cos ^{2} \phi}-\omega_{E}
\end{array}\right.
$$

where $\omega_{E}$ stands for the Earth angular velocity, i.e. $0.00007292115 \mathrm{rad} / \mathrm{sec}$.

The faster a satellite moves, the lower possibility for appearing at a station obtains. This is the rationale behind introducing our innovative method of the MEO satellites availability computation. To determine the Satellite Appearance Distribution Function (SADF) as the local visibility indicator of a satellite, we divide the mean sphere of the orbit into an equiangular $1^{\circ} \times 1^{\circ}$ grid. Each block is specified by its center point coordinates where for the $n m^{t h}$ block the coordinates $\left(\phi_{n}, \lambda_{m}\right)$ is defined as

$$
\begin{cases}\phi_{n}=n-0.5 & n=-89,-88, \cdots, 90 \\ \lambda_{m}=m-0.5 & m=1,2, \cdots, 360\end{cases}
$$

$D_{n m}$, the $n m^{\text {th }}$ local visibility number is

$$
D_{n m}= \begin{cases}\frac{k}{\sqrt{\omega_{\phi}^{r 2}+\omega_{\lambda}^{r^{2}}}} & -i \leq \phi_{n} \leq i \\ 0 & \text { elsewhere }\end{cases}
$$

It is a measure of the extent with which the $n m^{\text {th }}$ cell contributes to the number of satellites $\left(n_{\text {sat }}\right)$ at a station. Conceptually, it is very similar to redundancy number $\left(r_{i}\right)$ and degree of freedom $(d f)$ in the classical geodetic control networks. $r_{i}$ is the contribution of each observation to $d f$. Similarly, $D_{n m}$ expresses the $n m^{t h}$ cell's contribution to the number of visible satellites at a station. Inserting $\omega_{\phi}^{r}$ and $\omega_{\lambda}^{r}$ from eq. (5) into eq. (7) gives

$$
D_{n m}=\frac{T}{2 \pi} \begin{cases}k \frac{\cos \phi_{n}}{\sqrt{\left(1-\tau^{2}\right) \cos ^{2} \phi_{n}+\cos ^{2} i \tan ^{2} \phi_{n}-2 \tau \cos i}} & \left|\phi_{n}\right| \leq i \\ 0 \quad \text { elsewhere } & \end{cases}
$$

where $\tau=\frac{T \omega_{E}}{2 \pi}$ and $k$ is proportional to the total number of satellites $N_{\text {sat }}$, forming a navigation system

$$
k=\frac{0.017453292 N_{\text {sat }}}{T \sum_{n=-i+1}^{i} \frac{\cos \phi_{n}}{\sqrt{\left(1-\tau^{2}\right) \cos ^{2} \phi_{n}+\cos ^{2} i \tan ^{2} \phi_{n}-2 \tau \cos i}}} .
$$




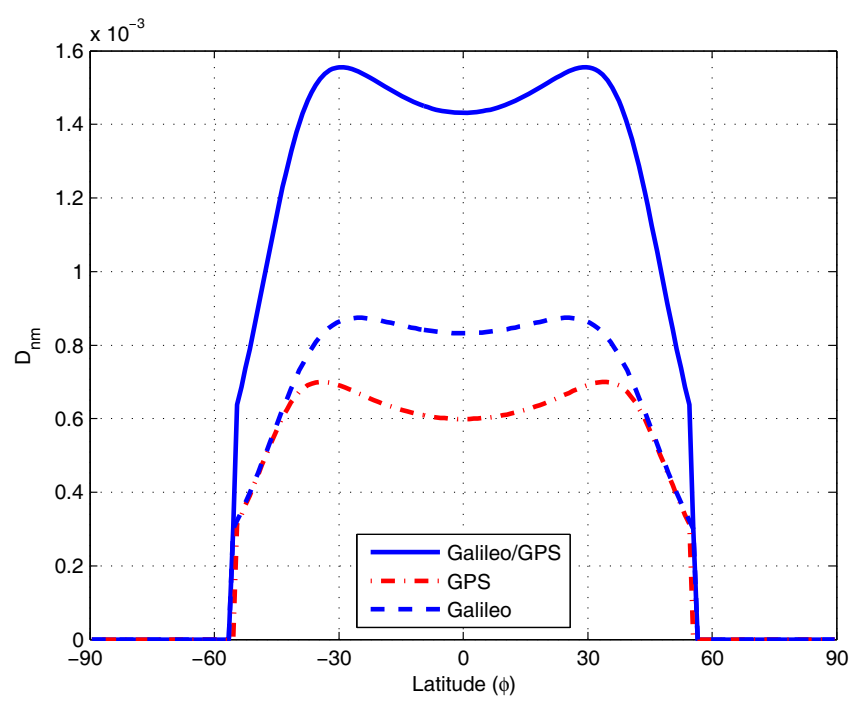

Figure 1: SADF versus geocentric latitude

One can compute the parameter for every positioning system simply by replacing the corresponding values for $N_{\text {sat }}, i$ and $\tau$. For instance, it is approximately equal to $5.27445437 \times$ $10^{-8}$ and $6.10642075 \times 10^{-8}$ for GPS and Galileo systems respectively. Therefore, the local visibility number of the $\mathrm{nm}^{\text {th }}$ block for a GPS satellite is

$$
D_{n m}=k_{G P S} \begin{cases}\frac{\cos ^{2} \phi_{n}}{\sqrt{\cos ^{4} \phi_{n}+\cos ^{2} 55 \sin ^{2} \phi_{n}}}\left|\phi_{n}\right| \leq 55^{\circ} \\ 0 \quad \text { elsewhere }\end{cases}
$$

whereas that of a Galileo satellite equals to

$$
D_{n m}=k_{\text {Galileo }}\left\{\begin{array}{l}
\frac{\cos ^{2} \phi_{n}}{\sqrt{\cos ^{4} \phi_{n}+\cos ^{2} 56 \sin ^{2} \phi_{n}}}\left|\phi_{n}\right| \leq 56^{\circ} \\
0 \quad \text { elsewhere }
\end{array}\right.
$$

SADF as a function of the geocentric latitude $\left(D_{n m}=D_{n} m(\phi)\right)$, for GPS, Galileo and a combined Galileo/GPS systems are plotted in Fig. (1).

As seen, the local visibility number of a Galileo satellite for the low and mid-latitude stations is higher than that of a GPS satellite. Moreover, the combination raises the number sharply.

\section{NUMBER OF SATELLITE VISIBLE AT A STATION (TOTAL VISIBIL- ITY NUMBER)}

When satellites are close to horizon, there is more atmosphere, troposphere, and ionosphere for the signals to go through. Consequently, the observable random error increases dramatically and vertical accuracy gets the worst. To prevent deficiency in positioning accuracy, we usually ignore the observed quantities to the satellites below the elevation mask angle. The angle determines the minimum elevation angle below which the receiver 
will no longer use a satellite in its computations. A typical value for the angle is $15^{\circ}$. Therefore, a satellite will be visible if its elevation is higher than the mask angle, i.e. $15^{\circ}$. The number of visible satellites $n_{p}$, available to a station $p$, on the Earth is calculated by accumulating $D_{n m}$ s for the satellites with zenith angle smaller than $75^{\circ}$

$$
\left\{\begin{array}{l}
n_{p}=\sum_{n=-89}^{90} \sum_{m=1}^{360} D_{n m} \\
\text { subject to } \\
Z_{p}^{n m} \leq 75^{\circ}
\end{array}\right.
$$

where, $Z_{p}^{n m}$ is the zenith angle defined by the station $p$ and the $n m^{\text {th }}$ block. Computation of $Z_{p}^{n m}$ would be then the first step. Let us consider an arbitrary station $p$ with $(L, B, h)$ coordinates in the conventional terrestrial system (CTS). Its Cartesian coordinates are (e.g. Keller 2001)

$$
\left(\begin{array}{l}
x_{p} \\
y_{p} \\
z_{p}
\end{array}\right)_{C T S}=\left(\begin{array}{c}
(N+h) \cos B \cos L \\
(N+h) \cos B \sin L \\
\left(N\left(1-e^{2}\right)+h\right) \sin B
\end{array}\right)
$$

where $N$ is the radius of curvature in the prime vertical

$$
N=\frac{a}{\sqrt{1-e^{2} \sin ^{2} B}} .
$$

$a$ and $e$ are the semi-major axis and the first numerical eccentricity of the utilized reference ellipsoid (WGS84).

With tagging the coordinates of each block onto the satellite appeared within the cell, we can write the satellite coordinates in terms the block coordinates. For instance, for the satellite appeared in the $n m^{\text {th }}$ block

$$
\left(\begin{array}{c}
X^{\text {sat }} \\
Y^{\text {sat }} \\
Z^{\text {sat }}
\end{array}\right)_{C I S}=\left(\begin{array}{c}
R \cos \phi_{n} \cos \lambda_{m} \\
R \cos \phi_{n} \sin \lambda_{m} \\
R \sin \phi_{n}
\end{array}\right)
$$

where $R$ is the mean radius of the orbital sphere. To provide a coherent coordinates, we transform satellites coordinates from the space-fixed equatorial system (CIS) to the conventional terrestrial system (CTS). The transition from CIS to CTS is substantiated through a sequences of rotations that accounts for precession, nutation and the Earth rotation including polar motion. These rotations can be expressed using a sequence of matrix multiplication (McCarthy, 1996)

$$
\left(\begin{array}{c}
x^{\text {sat }} \\
y^{\text {sat }} \\
z^{\text {sat }}
\end{array}\right)_{C T S}=\mathbf{P N}(t) \mathbf{R}(t) \mathbf{W}(t)\left(\begin{array}{c}
X^{\text {sat }} \\
Y^{\text {sat }} \\
Z^{\text {sat }}
\end{array}\right)_{C I S},
$$

where $P N(t), R(t)$ and $W(t)$ are the transformation matrices arising from the motion of the Celestial Ephemeris Pole (CEP) in the CIS, from the rotation of the Earth around the axis of the CEP, and from polar motion respectively. For our application, $P N(t)$ and $W(t)$ can be easily replaced by the identity matrix (I) without loss of generality 


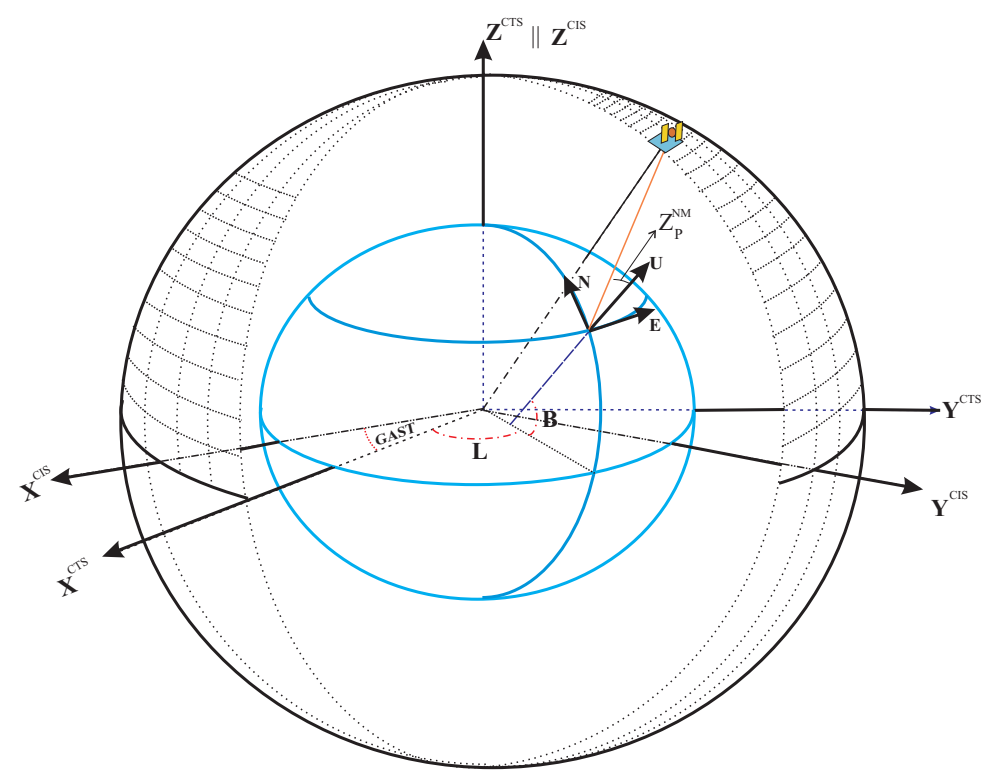

Figure 2: The utilized coordinate systems

$$
\left(\begin{array}{l}
x^{\text {sat }} \\
y^{\text {sat }} \\
z^{\text {sat }}
\end{array}\right)_{C T S}=\mathbf{R}_{3}(G A S T)\left(\begin{array}{c}
X^{\text {sat }} \\
Y^{\text {sat }} \\
Z^{\text {sat }}
\end{array}\right)_{C I S}
$$

where GAST is Greenwich Apparent Sidereal Time and $\mathbf{R}_{3}$ is the rotation matrix around the third axis $\left(Z^{C I S}\right)$.

Finally, for transformation of coordinate differences from CTS or the global ellipsoidal coordinate system to the Local Ellipsoidal Coordinate System (LES), denoted by $(N, E, U)$, we obtain the relation

$$
\left(\begin{array}{c}
U \\
E \\
N
\end{array}\right)_{L E S}=\mathbf{R}_{2}(-B) \mathbf{R}_{3}(L)\left(\begin{array}{l}
x^{s a t}-x_{p} \\
y^{s a t}-y_{p} \\
z^{s a t}-z_{p}
\end{array}\right)
$$

in which, $\mathbf{R}_{2}$ is the rotation matrix around the second axis of CTS $\left(y^{C T S}\right)$. The utilized coordinate systems are visualized in Fig. (2) altogether. Eventually, the zenith angle $Z_{p}^{n m}$ is calculated using eq. (19)

$$
Z_{p}^{n m}=\arccos \frac{U}{\sqrt{U^{2}+E^{2}+N^{2}}} .
$$

Combining eqs. (19) and (12) yields

$$
\left\{\begin{array}{l}
n_{p}=\sum_{n=-89}^{90} \sum_{m=1}^{360} D_{n m} \\
\text { subject to } \\
\frac{U}{\sqrt{U^{2}+E^{2}+N^{2}}} \leq \cos 75^{\circ}
\end{array}\right.
$$




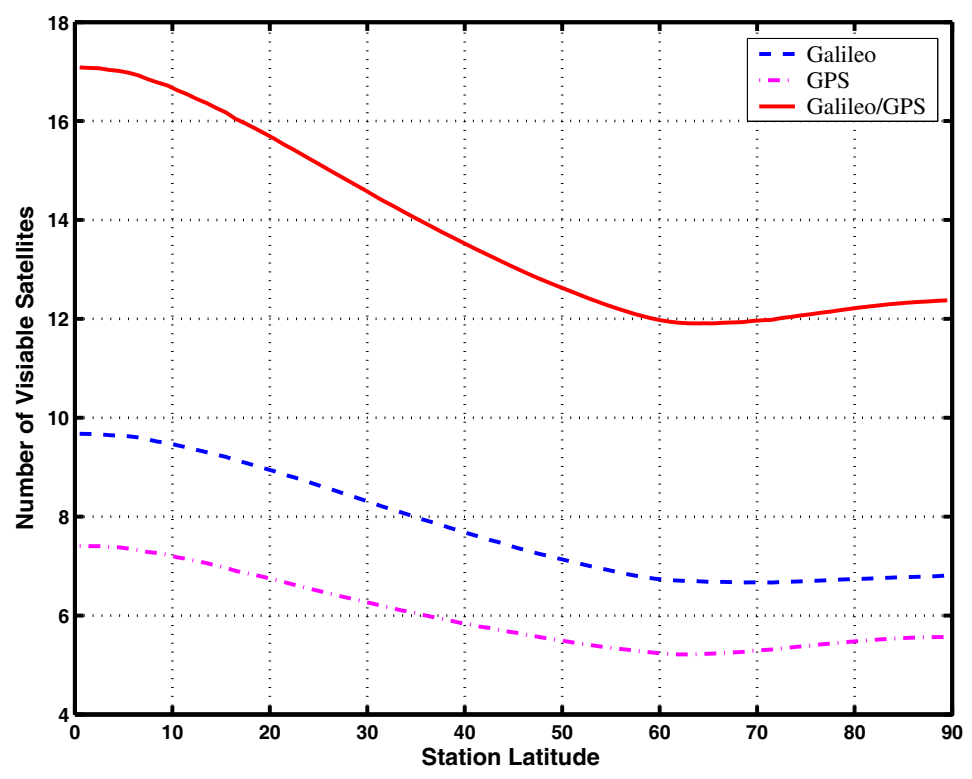

Figure 3: Number of available satellites at different geodetic latitudes

Solving eq. (20) for any desired station on the Earth results in the number of visible satellite at the station. We have considered GPS and Galileo systems respectively with 24 and 30 satellites which have been distributed with continuous distribution (Geiger, 1988). Fig. (3) shows the number of available satellites versus geodetic latitude.

As seen, in all cases the maximum number of satellites is accessible at the equator, whereas the number is minimum at latitude $60^{\circ}$. At least 4 GPS satellites are visible at any time and any location ( in the absence of the natural or man-made barriers), while this number is 6 for the Galileo system. Totally, at least 11 satellites of the kind GPS or Galileo can be tracked whenever and wherever a GPS/Galileo receiver is mounted. Due to symmetry of the diagram with respect to the equator, we only represent the visible satellites at the stations located on the northern hemisphere.

\section{LINEAR SYSTEM OF OBSERVATION EQUATIONS OF THE NAVIGA- TION SOLUTION}

Pseudo-range observable is common both to GPS and Galileo system. Eq. (21) expresses the observable in terms of satellite and station coordinates

$$
v+\rho_{p}^{n m}=\sqrt{\left(x^{s a t}-x_{p}\right)^{2}+\left(y^{s a t}-x_{p}\right)^{2}+\left(z^{s a t}-x_{p}\right)^{2}}-c \delta t,
$$

where $v$ is the observation's residual and $\rho_{p}^{n m}$ stands for the observed distance at the station $P$ to a satellite appeared in the $n m^{\text {th }}$ block. Synchronization error of the receiver and the space segment as well as the propagation error is expressed in $c \delta t$ (Geiger, 1988). Linearization of eq. (21) with respect to the station coordinates and clock error, as the unknowns leads to a linear observation equation of the type 


$$
v=\left(\begin{array}{llll}
-\frac{x^{s a t}-x_{p_{0}}}{\rho_{p_{0}}^{n m}} & -\frac{y^{s a t}-y_{p_{0}}}{\rho_{p_{0}}^{n m}}-\frac{z^{s a t}-z_{p_{0}}}{\rho_{p_{0}}^{n m}} & -1
\end{array}\right)\left(\begin{array}{c}
\delta x_{p} \\
\delta y_{p} \\
\delta z_{p} \\
c \delta t
\end{array}\right)-\left(\rho_{p}^{n m}-\rho_{p_{0}}^{n m}\right),
$$

in which, $\left(x_{p_{0}}, y_{p_{0}}, z_{p_{0}}\right)$ is the approximate coordinates of the station $P$ and $\rho_{p_{0}}^{n m}$ is the computed range using the approximate coordinates. For ease of representation, we rewrite the equation as

$$
v=\left(\begin{array}{llll}
e_{1} & e_{2} & e_{3} & -1
\end{array}\right)\left(\begin{array}{l}
\delta x_{p} \\
\delta y_{p} \\
\delta z_{p} \\
c \delta t
\end{array}\right)-\delta l
$$

$\left(e_{1}, e_{2}, e_{3}\right)$ is the unit vector pointed from the satellite to the station (Geiger, 1988). Considering the value of SADF as the weight of the observations to the satellite appeared in each cell gives the normal matrix of equations as follows

$$
\mathbf{N}_{4 \times 4}=\left(\begin{array}{cc}
\mathbf{N}_{\mathbf{1 1}}(3 \times 3) & \mathbf{N}_{12}(3 \times 1) \\
\mathbf{N}_{12}^{T}(1 \times 3) & \mathbf{N}_{22}(1 \times 1)
\end{array}\right)
$$

where

$$
\left\{\begin{array}{l}
\mathbf{N}_{11}(i, j)=\sum_{n=-89}^{90} \sum_{m=1}^{360} D_{n m} e_{i} e_{j} \\
\mathbf{N}_{12}(i, 1)=\sum_{n=-89}^{90} \sum_{m=1}^{360} D_{n m} e_{i} \\
\mathbf{N}_{22}=\sum_{n=-89}^{90} \sum_{m=1}^{360} D_{n m} \\
\text { subject to } \frac{U}{\sqrt{U^{2}+E^{2}+N^{2}}} \leq \cos 75^{\circ}
\end{array}\right.
$$

where $i$ and $j$ run over $\{1,2,3\}$. Inversion of the normal matrix yields variance-covariance matrix of the estimated parameters $\left(\delta x_{p}, \delta y_{p}, \delta z_{p}, c \delta t\right)$. We can derive inverse of the normal matrix with a similar structure using the partitioning formulae

$$
\mathbf{Q}_{\mathbf{x x}}=\mathbf{N}^{-1}=\left(\begin{array}{ll}
\mathbf{Q}_{11} & \mathbf{Q}_{12} \\
\mathbf{Q}_{12}^{T} & \mathbf{Q}_{22}
\end{array}\right)
$$

$\mathbf{Q}_{11}$ and $\mathbf{Q}_{22}$ are the estimated station coordinates and the receiver clock difference autocovariance matrices respectively. $\mathbf{Q}_{12}$ contains cross-covariances between the two sets of estimated parameters. Implementation of the partitioning relationships yields (e.g. Wells, 1994)

$$
\left\{\begin{array}{l}
\mathbf{Q}_{11}=\left(\mathbf{N}_{11}-\mathbf{N}_{12} \mathbf{N}_{22}^{-1} \mathbf{N}_{21}\right)^{-1} \\
\mathbf{Q}_{22}=\left(\mathbf{N}_{22}-\mathbf{N}_{21} \mathbf{N}_{11}^{-1} \mathbf{N}_{12}\right)^{-1} \\
\mathbf{Q}_{12}=-\mathbf{N}_{11}^{-1} \mathbf{N}_{12} \mathbf{Q}_{22}
\end{array}\right.
$$




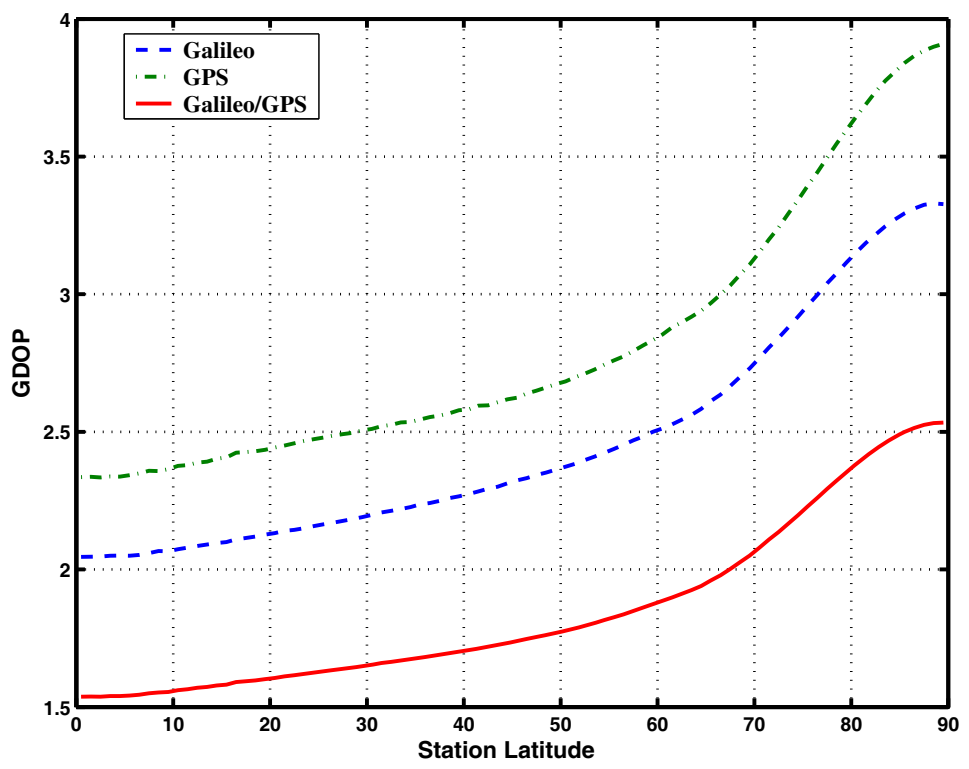

Figure 4: GPS, Galileo and Galileo/GPS GDOPs at different geodetic latitudes

The covariance matrix contains a part of the accuracy of satellite positioning which depends on the geometric configuration of the employed satellites. The concealed information in the matrix is frequently used in satellite positioning and called Dilution Of Precision (DOP). For instance, the combined effect for position and time called GDOP, is calculated as follows (Seeber, 2003)

$$
G D O P=\sqrt{\operatorname{trace}\left(\mathbf{Q}_{\mathbf{x x}}\right)}
$$

GDOP is calculated for GPS, Galileo and Galileo/GPS combination. The achieved results are plotted in Fig. (4) versus geodetic latitude.

Obviously, stations located at the Equator experience the best GDOP in all solutions while the near polar stations go through the worst. Galileo and GPS combination will sharply decrease the quantity thoroughly.

We can easily decompose GDOP into PDOP and TDOP. They hold the following relationship (Yarlagadda et al., 2000)

$$
G D O P=\sqrt{(P D O P)^{2}+(T D O P)^{2}} .
$$

Where $P D O P$ and TDOP are two different DOP designations are in use for $3 D$ positioning and time determination respectively. In terms of variance-covariance matrix entries, they can be defined as

$$
P D O P=\sqrt{\operatorname{trace}\left(\mathbf{Q}_{11}\right)}
$$

and

$$
T D O P=\sqrt{\mathbf{Q}_{22}}
$$



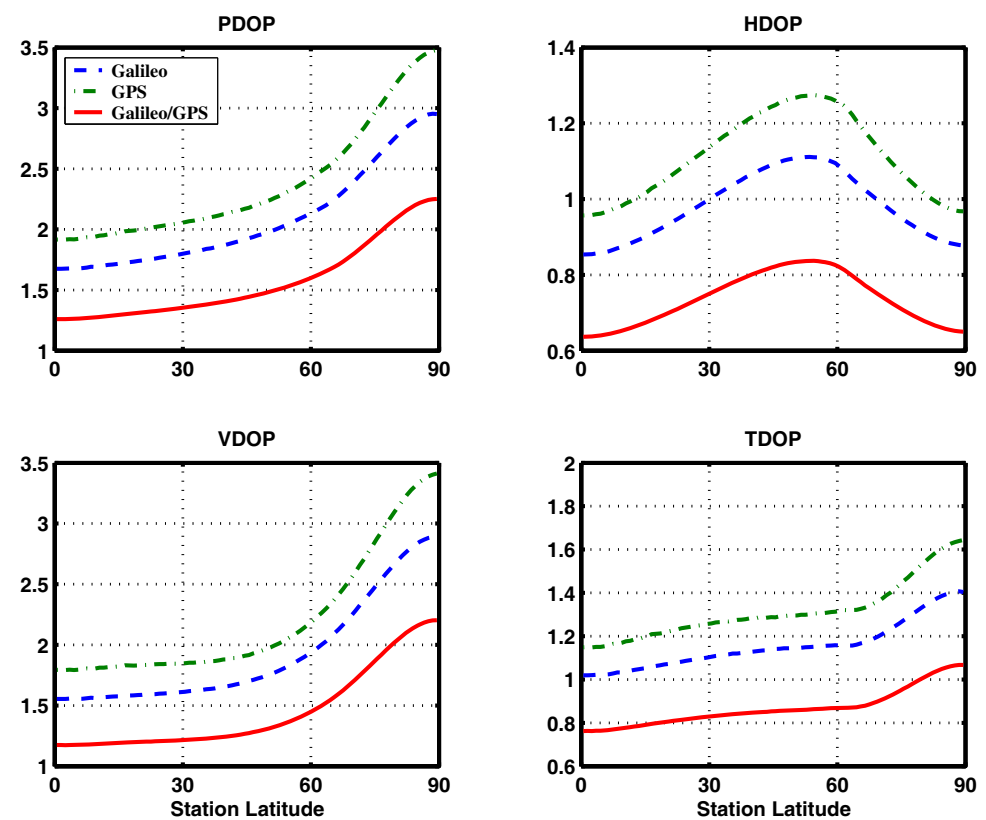

Figure 5: PDOP, TDOP, VDOP and $H D O P$ at different geodetic latitudes

Similarly, $P D O P$ can be split up into horizontal and vertical components, denoted by $H D O P$ and $V D O P$. Their representations in the local coordinate system, i.e. LES is preferred. Therefore, we calculate variance-covariance matrix of UEN coordinates using the propagation law to derive explicit expressions for the latest defined forms of DOP

$$
\mathbf{Q}_{U E N}=\mathbf{R}_{2}(-B) \mathbf{R}_{3}(L) \mathbf{Q}_{11} \mathbf{R}_{3}(-L) \mathbf{R}_{2}(B) .
$$

As already mentioned, $E$ and $N$ defines the local horizontal plane of the station while $U$ points to the zenith. Using $\mathbf{Q}_{U E N}$, dilution of precision are then derived for horizonal and vertical positioning

$$
\left\{\begin{array}{l}
V D O P=\sqrt{\mathbf{Q}_{U E N}(1,1)} \\
H D O P=\sqrt{\mathbf{Q}_{U E N}(2,2)+\mathbf{Q}_{U E N}(3,3)}
\end{array}\right.
$$

These two quantities reflect the utilized satellites geometrical constellation contributions to the horizontal and vertical accuracies. PDOP, TDOP, VDOP and HDOP are plotted for stations with different latitudes in Fig. (5).

Like $G D O P$, the equatorial stations have the minimum value for $P D O P, V D O P$ and $T D O P$ for all three solutions whereas the near polar stations will experience the maximum values. $H D O P$ shows a completely different pattern of error. It will reach its maximum at the stations with latitude around $55^{\circ}, \phi=i$.

Furthermore, $\mathbf{Q}_{U E N}$ contains information on the horizontal components of the station position in N-S and E-W directions. For instance, plotting the ratio of the third and second 


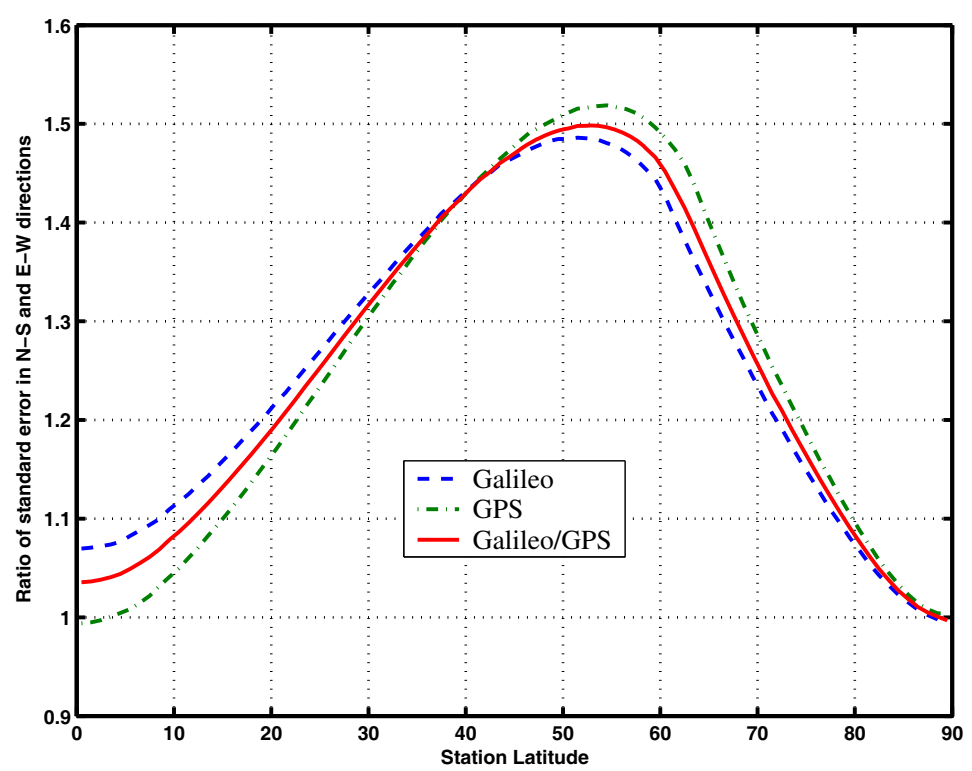

Figure 6: Ratio of $\mathrm{N}$ and $\mathrm{E}$ components versus the station latitude

diagonal elements $\left(\frac{\mathbf{Q}_{U E N}(3,3)}{\mathbf{Q}_{U E N}(2,2)}\right)$ at different latitudes shows fluctuation of the components accuracy with station latitude variation, see Fig. (6).

As seen, the components have nearly the same accuracy at the polar and equatorial stations. While the E-component is determined with higher accuracy at the mid-latitude stations with respect to the other.

This behavior can be interpreted by considering the satellites' geometrical configuration. $\phi=i$ is the turning point of the satellites, so the cells with $\phi=i$ are surveyed just in the $\mathrm{E}-\mathrm{W}$ direction $\left(\omega_{\phi}^{r}=0\right)$. Therefore, position of the stations at $\phi=i$ are more likely determined using the satellites located at the same latitude. Consequently, the N-component is determined rather weakly compared to the E-component. This interpretation is valid for three solutions.

Indeed, we compute cross-covariance matrix of the local coordinates and clock difference parameter to figure out time determination and positioning uncertainties correlation arising from satellites constellation. Implementation of the propagation law yields

$$
\mathbf{C}_{U E N t}=\left(\begin{array}{cc}
\mathbf{C}_{U E N} & \mathbf{C}_{U E N, t} \\
\mathbf{C}_{t, U E N} & \mathbf{C}_{t}
\end{array}\right)=\left(\begin{array}{cc}
\mathbf{S Q}_{11} \mathbf{S}^{T} & \mathbf{S Q}_{12} \\
\mathbf{S Q}_{12}^{T} & \mathbf{Q}_{22}
\end{array}\right)
$$

where $\mathbf{S}$ is the transformation matrix from CTS to UEN. Hence,

$$
\mathbf{S}=\mathbf{R}_{2}(-B) \mathbf{R}_{3}(L)
$$

$\mathbf{C}_{U E N, t}$ is comprised of cross-covariances of the coordinates and clock difference. For instance, $\mathbf{C}_{U E N, t}(1,1)$ represents vertical positioning and time determination covariance. 


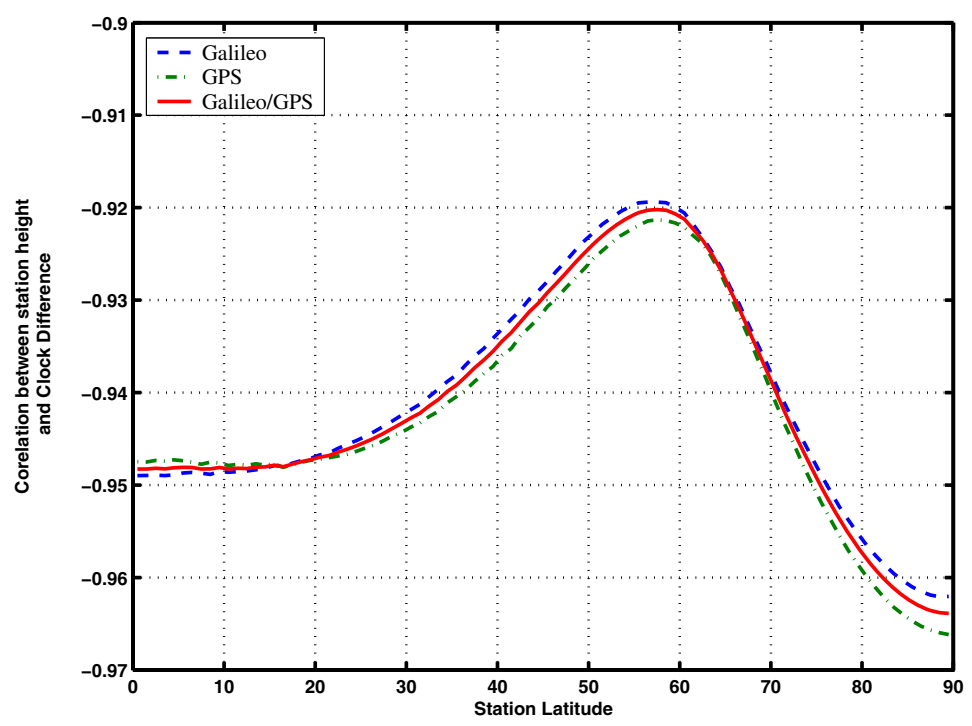

Figure 7: Correlation coefficient of the station height and clock difference

To get a dimensionless indicator, we compute the corresponding correlation coefficient

$$
\rho_{u, t}=\frac{\mathbf{C}_{U E N, t}(1,1)}{\sqrt{\mathbf{C}_{U E N}(1,1)} \sqrt{\mathbf{C}_{t}}} .
$$

The achieved results for the correlation coefficient at different latitudes are depicted in Fig. (7).

As it is seen, they are strongly correlated to each other, so that the different solutions coefficients (absolute values) reach their minimum (approximately 0.92 ) around $\phi=60^{\circ}$. From statistical point of view, even for the other latitudes the coefficients reflect a very strong correlation between the two estimated parameters. This correlation is eminently reasonable because the vertical positioning is directly affected by time determination error. Moreover, the negative sign of the coefficient is due to inverse proportionality of the station height and the travel time of the emitted signal from the satellites to the station.

\section{CONCLUSIONS}

The US government has monopoly on satellite based navigation because of military authorities of GPS. Even though GLONASS, the Russian GNSS, was developed simultaneously, it has only few users due to its deficiency. It is going to be over by launching a new generation of GNSS under civil authorities. The new GNSS called Galileo is managed by the European Union and the European Space Agency.

To compare the coming system with the preceding one, i.e. GPS, performance parameters are used. In the context of GNSS, availability, accuracy, integrity and continuity are frequently used as the performance measures. Among them, availability, the number 
of satellites available to the user, investigated in this paper. We introduced an innovative approach for the MEO satellites availability computation. Besides developing the mathematical formulation of the scheme, different achievable accuracy using GPS, Galileo and a combined Galileo/GPS compared numerically.

Continuous satellite distributions were considered for simulation both for GPS and Galileo system and the numerical analysis carried out using the simulated data. The achieved results for GPS, Galileo, and Galileo/GPS in terms of different designations of DOP were compared. From constellation point of view, Galileo is superior to GPS because of offering relatively higher accuracy nearly in all instances. We also showed that a combined GPS and Galileo system sets up a robust and reliable satellite navigation and positioning system of extraordinary performance, as discussed in (Meng et al., 2003).

\section{REFERENCES}

Geiger A. (1988). Simulating disturbances in GPS by continuous satellite distribution, Journal of Surveying Engineering, 114, 182-194.

Keller W. (2001). Geodetic coordinate systems, Lecture notes, Department of geodesy and geoInformatics, University of Stuttgart.

Krakiwsky E. J., D. B. Thomson (1974) Geodetic position computations, Lecture notes, No. 39, Department of geodesy and geomatics engineering, University of New Brunswick, Fredericton.

McCarthy D. (1996). IERS conventions (1996), IERS technical notes No. 21, International Earth Rotation Service.

Meng X., C. Noakes, A. H. Dodson and G. W. Roberts (2003). GPS satellite geometry and its implications for structural deformation monitoring, ION 2003 proceedings, 9-12.

O'Keefe K., S. G. Ryan and G. Lachapelle (2002). Global availability and reliability assessment of the GPS and Galileo global navigation satellite systems, Canadian Aeronautics and Space J., 48, 123-132.

Santerre R. (1991). Impact of GPS satellite sky distribution, manuscripta geodetica, 16, 28-53.

Seeber G. (2003). Satellite Geodesy: Foundations, Methods \& Applications, Walter de Gruyter, Berlin.

U.S. Department of Transportation and U. S. Department of Defense (2001). Federal Radionavigation Systems, Final Report, DOT/VNTSC/RSPA/01-3.1/DoD-4650.5.

Wang J., H. I. Bki and L. Caiping (2002). Dependency of GPS positioning precision on station location, GPS Solutions, 6, 91-95.

Wells D. (1994). Functional analysis applied to least squares in geomatics, Lecture notes, Department of geodesy and geomatics engineering, University of New Brunswick, Fredericton.

Wolfrum J., and H. Trautenberg (2000). Galileo - a technical concept for the European satellite navigation system, Int. J. Satell. Commun., 18, 243-258.

Yarlagadda R., I. Ali, N. Al-Dhahir and J. Hershey (2000). GPS GDOP metric, IEE Proceedings on Radar, Sonar, and Navigation, 259-264.

Received: 2005-10-13,

Reviewed: 2006-01-06,

Accepted: 2006-02-27. 